\title{
THE VECTOR LATTICE COVER OF CERTAIN PARTIALLY ORDERED GROUPS
}

\author{
G. BUSKES and A. VAN ROOIJ
}

(Received 24 January 1991)

Communicated by P. G. Dodds

\begin{abstract}
In this paper we introduce the notion of Riesz homomorphism on Archimedean directed partially ordered groups and use it to study the vector lattice cover of such groups.
\end{abstract}

1991 Mathematics subject classification (Amer. Math. Soc.): 46 A 40, 06 F 15.

There are close relations between the theories of Boolean algebras, distributive lattices, abelian lattice ordered groups and the like on one hand and vector lattices (= Riesz spaces) on the other hand. In the case of Boolean algebras such a relation is made explicit by considering the elements of a Boolean algebra as so called place functions. The set of these place functions generates a vector lattice and this vector lattice can then be used to study the Boolean algebra (see Fremlin's book [8]).

In the other cases mentioned, one simply "forgets" some of the structure of a Riesz space if one considers it as a lattice ordered group or a distributive lattice. Conrad in [6] introduced the notion of a vector lattice cover for Archimedean lattice ordered groups and indeed, in categorical language, he therefore studied "the adjoint of the forgetful functor". Shortly thereafter, Bleier in [3] proved a somewhat more general result than Conrad's: For every Archimedean lattice ordered group $G$ there exists an (essentially) unique Archimedean Riesz space $E(G)$ that contains $G$ as a lattice ordered subgroup and which is minimal with that property; furthermore, such a $G$ is automatically large in $E(G)$ (that is, for every $0<g \in E(G)$ there exists

(C) 1993 Australian Mathematical Society 0263-6115/93 $\$ A 2.00+0.00$ 
$f \in G$ and $n \in \mathbb{N}$ such that $0<f \leq n g$ ). There is, in various circumstances, a need to have such a vector lattice cover for Archimedean directed partially ordered groups. There is an abundance of examples in the literature where such a notion may be applied effectively. We mention the selfadjoint operators on a real Hilbert space, the regular operators between two vector lattices, the vector space tensor product of two vector lattices, a vector space with the ordering induced by the free vector lattice that it generates [1], the relatively central operators [11].

In this paper we develop the notion of a vector lattice cover for integrally closed directed partially ordered groups. Most of the technical problems stem from the fact that there is not readily available the notion of "morphism". Nonetheless, we introduce such a notion and hasten to say that it does not act in a categorical fashion (see 1.9 and 1.10). It therefore seems that our results do not follow from that more abstract machine, called category theory. Our results extend those of Conrad and Bleier [3], [6], [7]. Our paper is in ZF set theory. For terminology we refer to [2].

\section{Riesz homomorphisms}

In this paper we intend to study a rather wide class of partially ordered structures from the viewpoint of the much narrower class of Riesz spaces. To make this study possible we need a substitute in the wider setting for the morphisms in the class of Riesz spaces. Because we were not able to find such a notion in the literature we introduce it here. For a subset $A$ of a partially ordered set we denote by $A \uparrow$ the set of upper bounds of $A$. Let $G$ and $H$ be partially ordered groups. We call an additive map $\phi: G \rightarrow H$ a Riesz homomorphism [complete Riesz homomorphism] if for every nonempty set $A$ in $G$ with not more than two elements [respectively every nonempty set $A$ in $G$ which is bounded from above] $\phi(A \uparrow)$ and $\phi(A) \uparrow$ have the same lower bounds.

Thus an additive map $\phi: G \rightarrow H$ is a Riesz homomorphism if for all $a$ and $b$ in $G$ the sets $\{y: y \in H, y \geq \phi(a), y \geq \phi(b)\}$ and $\{\phi(x): x \in$ $G, x \geq a, x \geq b\}$ have the same lower bounds. By taking $a=b=0$ we see that this implies $0(\in H)$ is a lower bound of $\phi\left(G^{+}\right)$, that is, that $\phi$ is increasing. We now discuss some particular cases. If $H$ is a lattice, then the set $\{y: y \in H, y \geq \phi(a), y \geq \phi(b)\}$ has a greatest lower bound, $\phi(a) \vee \phi(b)$. Thus, if $H$ is a lattice, then an additive $\phi: G \rightarrow H$ is a Riesz homomorphism if and only if

$$
\phi(a) \vee \phi(b)=\inf \{\phi(x): x \in G, x \geq a \text { and } x \geq b\} .
$$


If both $G$ and $H$ are lattices, then the Riesz homomorphisms $G \rightarrow H$ are just the group homomorphisms that are also lattice homomorphisms. In particular, if $G$ and $H$ are Riesz spaces our notion of "Riesz homomorphism" coincides with the usual one. More generally, if $G$ is an ordered vector space and $H$ is a Riesz space, our "Riesz homomorphisms" coincide with the objects studied in $[9,1.8 .1]$. The " $R$-homomorphisms' studied in $[10]$ are another class of examples for our "Riesz homomorphisms". Furthermore, if $G$ and $H$ are Riesz spaces our "complete Riesz homomorphisms" coincide with the order continuous Riesz homomorphisms. The reader is warned that some operations that one is used to in dealing with Riesz homomorphisms may no longer hold in this more general setting. In particular, one has to be careful with restrictions and compositions of Riesz homomorphisms. That is, these may no longer be Riesz homomorphisms.

In this section we state and prove those facts about Riesz homomorphisms which play a role in understanding the notion of an enveloping Riesz space. We first show a very convenient reformulation of the concept. For nonempty subsets $A, B$ of $G$ and we denote $B-A=\{b-a: b \in B, a \in A\}$. If $G$ is integrally closed and $A \subset G$ is nonemtpy and bounded above then $\inf (A \uparrow-A)=0$ (Proof: of course, $A \uparrow-A \subset G^{+}$. On the other hand, let $u \in G$ be a lower bound of $A \uparrow-A$. Then for every $b \in A \uparrow, b-u$ is an upper bound of $A$, that is, $b-u \in A \uparrow$. From the fact that $G$ is integrally closed and the conditions on $A$ it follows that $u \leq 0$ ). In the sequel $G$ and $H$ are integrally closed directed partially ordered groups. We remark that by [2, Theorem 24, p. 312] the Dedekind completion of $G$ and $H$ by nonvoid cuts, $G^{\delta}$ and $H^{\delta}$ respectively, are groups.

LeMmA 1.1. Let $\phi: G \rightarrow H$ be additive.

(i) $\phi$ is a Riesz homomorphism if and only if for all $a, b \in G$ and $A=$ $\{a, b\}, \inf \phi(A \uparrow-A)=0$.

(ii) $\phi$ is a complete Riesz homomorphism if and only if for all $X \subset G$ with $\inf X=0$ we have inf $\phi(X)=0$.

Proof. (i) Suppose $\phi$ is a Riesz homomorphism.

Let $a, b \in G, A=\{a, b\}, B=\{\phi(a), \phi(b)\}$. Take a lower bound $u$ of $\phi(A \uparrow-A)$ in $H$; we wish to prove that $u \leq 0$. Now $u+\phi(a)$ and $u+\phi(b)$ are lower bounds of $\phi(A \uparrow)=\{\phi(x): x \in G, x \geq a, x \geq b\}$, hence are lower bounds of $\{y \in H: y \geq \phi(a), y \geq \phi(b)\}=B \uparrow$. Thus, if $y \in B \uparrow$, then $y-u \in B \uparrow$. As $B \uparrow$ is bounded from below and $H$ is integrally closed, we have $u \leq 0$.

Conversely, suppose $\inf \phi(A \uparrow-A)=0$ for every nonempty $A \subset G$ with not more than two elements. By taking $A=\{0\}$, we see that $\phi$ is increasing. 
Let $a, b \in G, A=\{a, b\}$. We wish to prove that $\phi(A) \uparrow$ and $\phi(A \uparrow)$ have the same lower bounds; then $\phi$ will be a Riesz homomorphism. As $\phi$ is increasing, $\phi(A \uparrow) \subset \phi(A) \uparrow$, so that every lower bound of $\phi(A) \uparrow$ is one of $\phi(A \uparrow)$. Conversely, let $u$ be a lower bound of $\phi(A \uparrow)$. For all $v \in \phi(A \uparrow)$, $u-v$ is a lower bound of $\phi(A \uparrow)-\phi(A)=\phi(A \uparrow-A)$, so $u-v \leq 0$ and $u \leq v$. Hence $u$ is a lower bound of $\phi(A) \uparrow$.

(ii) In precisely the same way one proves that $\phi$ is a complete Riesz homomorphism if and only if

$$
\inf \phi(A \uparrow-A)=0
$$

for every nonempty $A \subset G$ that is bounded from above. Hence, the condition

$$
X \subset G, \quad \inf X=0 \Rightarrow \inf \phi(X)=0
$$

implies that $\phi$ is a complete Riesz homomorphism.

To prove the reverse implication, let $X \subset G$ be such that inf $X=0$. Put $A=\{-x: x \in X\}$; then $A \uparrow-A=G^{+}+X$. Assuming that $\phi$ is a complete Riesz homomorphism, we see that $\phi$ is increasing, so that $\phi(X) \subset H^{+}$and inf $\phi\left(G^{+}+X\right)=0$. Consequently, inf $\phi(X)=0$.

Lemma 1.1 has some interesting corollaries.

Corollary 1.2. The natural map of $G$ into its Dedekind completion is a complete Riesz homomorphism.

Corollary 1.3. Let $\phi: G \rightarrow H$ be a Riesz homomorphism and let $\omega: H \rightarrow J$ be a complete Riesz homomorphism. Then $\omega \circ \phi$ is a Riesz homomorphism. If $\phi$ is complete, then so is $\omega \circ \phi$.

Corollary 1.4. Let $\phi: G \rightarrow H$ be a [complete] Riesz homomorphism; let $H_{0}$ be a directed subgroup of $H$ that contains $\phi(G)$. Then $\phi$, considered as a map $G \rightarrow H_{0}$, is a [complete] Riesz homomorphism.

Corollary 1.5. Let $\phi: G \rightarrow H$ be a group homomorphism, $\phi^{\delta}$ the induced group homomorphism $G \rightarrow H^{\delta}$. Then $\phi$ is a [complete] Riesz homomorphism if and only if $\phi^{\delta}$ is.

Corollary 1.6. For every $p \in \mathbb{N}$ the map $x \rightarrow p x$ of $G$ into $G$ is a complete Riesz homomorphism.

CoROllaRy 1.7. Let $\phi: G \rightarrow H$ be a Riesz homomorphism and suppose $G$ is a lattice. Then $\phi(G)$ is a lattice: if $a, b \in \phi(G)$ then $a \vee_{\phi(G)} b=a \vee_{H} b$. 
Corollary 1.8. Let $\phi: G \rightarrow H$ be a Riesz homomorphism. Then the natural maps $G \rightarrow \phi(G)$ and $\phi(G) \rightarrow H$ are Riesz homomorphisms.

The proofs of Corollary 1.2 through 1.5 are standard. We will prove Corollary 1.6, which is somewhat more involved, below. For Corollary 1.7 remark that $\phi^{\delta}: G \rightarrow H^{\delta}$ is a homomorphism of lattice groups. Corollary 1.8 is proved similarly.

Proof of Corollary 1.6. Let $X \subset G$, inf $X=0$ and $p \in \mathbb{N}$. Let $\mathscr{P}$ be the set of all finite subsets of $X$ ordered by inclusion. Defining

$$
y_{\alpha}=\inf _{x \in \alpha} \delta(x) \quad(\alpha \in \mathscr{P})
$$

where $\delta$ is the natural map $G \rightarrow G^{\delta}$, we obtain a decreasing net $\left(y_{\alpha}\right)_{\alpha \in \mathscr{P}}$ in $G^{\delta}$. We have $\inf X=0$, hence by Corollary $1.2 \inf \delta(X)=0$, so that $y_{\alpha} \downarrow 0$. It follows from [2, Theorem 26, p. 314] that $p y_{\alpha} \downarrow 0$. Consequently, $\inf p \delta(X)=0$. Then inf $p X=0$.

EXAMPLE 1.9. The restriction of a Riesz homomorphism may fail to be a Riesz homomorphism.

Let $G=C[0,1]$. The polynomial functions on $[0,1]$ of degree $\leq 1$ form a linear subspace $D$ of $G . D$ is integrally closed since $G$ is Archimedean and $D$ is directed. $\omega: f \rightarrow f(1 / 2)$ is a Riesz homomorphism $G \rightarrow \mathbb{R}$ but its restriction to $D$ is not a Riesz homomorphism.

EXAMPLE 1.10. The composition of two Riesz homomorphisms may fail to be a Riesz homomorphism.

Let $G=C[-1,1], D=\{x \in C[-1,1]: 2 x(0)=x(1)+x(-1)\} . \quad D$ is order dense in $G$. It follows that the natural map $\phi: D \rightarrow G$ is a complete Riesz homomorphism. $\omega: G \rightarrow \mathbb{R}$ defined by $\omega(f)=f(0)$ is a Riesz homomorphism, but $\omega \circ \phi$ is not. (Let $x \in C[-1,1]$ be the identity function of $[-1,1]$. Take $a=1+x, b=1-x$. If $x \in D, x \geq a$ and $x \geq b$ then $x(1) \geq a(1)=2$ and $x(-1) \geq b(-1)=2$, so

$$
x(0) \geq 1 / 2(x(1)+x(-1))=2>a(0) \vee b(0) .)
$$

Before continuing with some positive news, one more piece of bad behaviour.

EXAMPLE 1.11. Let

$G=\left\{x \in l^{\infty}(\mathbb{Z}): \lim _{|n| \rightarrow \infty} x(n)=x(0), \quad x(n)+x(-n)=2 x(0)\right.$ for every $\left.n\right\}$, $H=c$ and $\phi: G \rightarrow H$ defined by $\phi(x)=\left.x\right|_{\mathbb{N}}(x \in G)$. Then $\phi$ is a bijective Riesz homomorphism $G \rightarrow H$ but not an order isomorphism. 
Proof. To see that $\phi$ is a Riesz homomorphism:

Take $a \in G$; we wish to show $(\phi(a))^{+}=\inf \left\{\phi(x): x \in G^{+}, x \geq a\right\}$. For $N \in \mathbb{N}$ define $x_{N}: \mathbb{Z} \rightarrow \mathbb{R}$ by

$$
x_{N}(n)= \begin{cases}a(n)^{+} & \text {if } n=1, \ldots, N \\ 2\|a\|_{\infty}-a(-n)^{+} & \text {if } n=-1, \ldots,-N \\ \|a\|_{\infty} & \text { if } n=0 \text { or }|n|>N .\end{cases}
$$

Then $x_{N} \in G^{+}, x_{N} \geq a$ for every $N$, whereas in $c$ we have $\left.\inf _{N} x_{N}\right|_{N}=$ $\left(\left.a\right|_{N}\right)^{+}$.

However, if $\phi: G \rightarrow H$ is bijective Riesz homomorphism and $G$ is a lattice, then $\phi$ is an order isomorphism.

(If $x \in G, x \not 0$, then $x^{+} \neq 0$, so $(\phi(x))^{+}=\phi\left(x^{+}\right) \neq 0$ and $\phi(x) \not 0$.)

\section{The enveloping Riesz space}

In this section we will develop the notion of enveloping Riesz space $E(G)$ for an integrally closed directed po-group $G$. It is not very complicated to construct $E(G)$ right away, but we have in mind to investigate primarily its universal properties. For the latter reason we start with a study of extending Riesz homomorphisms.

Definition 2.1. $\phi: G \rightarrow H$ is a $V$-homomorphism if for all $a, b \in G$ the sets $\{y \in H: y \geq \phi(a)$ and $y \geq \phi(b)\}$ and $\{\phi(x): x \in G, x \geq a, x \geq b\}$ have the same lower bounds. Thus, a Riesz homomorphism is an additive V-homomorphism. If $G$ and $H$ are Riesz spaces our definition coincides with the one in [4]. The motivation for the next lemma is [4].

Lemma 2.2. Let $E$ be a vector space, $F$ a $\sigma$-Dedekind complete Riesz space, $p: E \rightarrow F$ a sublinear map and $a$ an element of $E$.

(i) The formula

$$
p^{a}(x)=\inf _{\mu \in \mathbb{R}} p(x+\mu a)-\mu p(a) \quad(x \in E)
$$

defines a sublinear $p_{a}: E \rightarrow F$. We have

$$
\begin{gathered}
-p(-x) \leq p_{a}(x) \leq p(x) \quad(x \in E), \\
p^{a}(a)=a, \quad p^{a}(-a)=-p(a)
\end{gathered}
$$

and even

$$
p^{a}(x+\lambda a)=p^{a}(x)+\lambda p(a) \quad(x \in E, \lambda \in \mathbb{R}) .
$$


(ii) If $D_{0}$ is a linear subspace of $D$ and $p$ is linear on $D_{0}$, then $p^{a}$ coincides with $p$ on $D_{0}$ and is linear on $D_{0}+\mathbb{R} a$.

(iii) Let $E$ be a directed vector space. If $p$ is a V-homomorphism, then so is $p^{a}$.

Proof. (i) For every $x \in D$ the map $\mu \rightarrow p(x+\mu a)-\mu p(a)$ of $\mathbb{R}$ into $F$ is decreasing and bounded from below by $-p(-x)$. It follows that we can define $p^{a}: E \rightarrow F$ by (1) and that (2) holds. (4) and (3) follow from the observation that for all $x$ and $\lambda$

$$
\begin{aligned}
p^{a}(x+\lambda a) & =\inf _{\mu} p(x+\lambda a+\mu a)-\mu p(a) \\
& =\inf _{\nu} p(x+\nu a)-(\nu-\lambda) a=p^{a}(x)+\lambda a .
\end{aligned}
$$

(ii) Apply (2) and then (4).

(iii) For every $\mu$ the map $x \rightarrow p(x+\mu a)-\mu p(a)$ is a $\vee$-homomorphism. The pointwise infimum of a decreasing net of $V$-homomorphisms is a $V$ homomorphism.

Lemma 2.3. Let $E$ be an Archimedean, directed partially ordered vector space, $G$ a subgroup of $E$ that generates $E$ as a vector space. Let $\phi$ be an order preserving group homomorphism of $G$ into an Archimedean directed partially ordered vector space $F$. Then $\phi$ extends uniquely to an order preserving linear map $\bar{\phi}: E \rightarrow F$. If $\phi$ is a Riesz homomorphism, then so is $\bar{\phi}$.

Proof. (I) We first show that $\mathbb{Q} G$ is relatively uniformly dense in $E$ in the following sense:

Let $x \in E$. Then there exists an $a \in G$ such that for every $\varepsilon>0$ there is $a y \in G$ with $y-\varepsilon a \leq x \leq y+\varepsilon a$.

Indeed, $x=\sum_{i=1}^{N} \lambda_{i} x_{i}$ for certain $\lambda_{1}, \ldots, \lambda_{N} \in \mathbb{R}, x_{1}, \ldots, x_{N} \in G$. Choose $a \in \mathbb{Q}$ such that

$$
a \geq x_{i} \text { and } a \geq-x_{i} \quad(i=1, \ldots, N) \text {. }
$$

(Then $a \geq 0$ ). Let $\varepsilon>0$. Take $\mu_{1}, \ldots, \mu_{N} \in \mathbb{Q}$ for which

$$
\left|\mu_{i}-\lambda_{i}\right| \leq \varepsilon \quad(i=1, \ldots, N) .
$$

Then for each $i$,

$$
\begin{aligned}
\mu_{i} x_{i} & =\lambda_{i} x_{i}+\varepsilon\left(-x_{i}\right)+\left(\mu_{i}-\lambda_{i}+\varepsilon\right) x_{i} \\
& \leq \lambda_{i} x_{i}+\varepsilon a+\left(\mu_{i}-\lambda_{i}+\varepsilon\right) a \leq \lambda_{i} x_{i}+3 \varepsilon a
\end{aligned}
$$

and similarly $\lambda_{i} x_{i} \leq \mu_{i} x_{i}+3 \varepsilon a$. Setting $y=\sum_{i} \mu_{i} x_{i}$ we obtain $y-3 N \varepsilon a \leq$ $x \leq y+3 N \varepsilon a$. 
(II) Now the lemma. We have to show: If $\lambda_{1}, \ldots, \lambda_{N} \in \mathbb{R}, x_{1}, \ldots, x_{N} \in$ $G$ and $\sum \lambda_{i} x_{i} \geq 0$ in $E$, then $\sum \lambda_{i} \phi\left(x_{i}\right) \geq 0$ in $F$. This we do as follows. Take $a$ as in (I). Let $\varepsilon>0, \varepsilon \in \mathbb{Q}$. Choose $\mu_{1}, \ldots, \mu_{N} \in \mathbb{Q}$ with $\left|\mu_{i}-\lambda_{i}\right| \leq$ $\varepsilon / 3 N$ for each $i$. By (I),

$$
\sum \mu_{i} x_{i}-\varepsilon a \leq \sum \lambda_{i} x_{i} \leq \sum \mu_{i} x_{i}+\varepsilon a
$$

and, similarly,

$$
\sum \mu_{i} \phi\left(x_{i}\right)-\varepsilon \phi(a) \leq \sum \lambda_{i} \phi\left(x_{i}\right) \leq \sum \mu_{i} \phi\left(x_{i}\right)+\varepsilon \phi(a) .
$$

Take $p \in \mathbb{N}$ such that $p \mu_{1}, \ldots, p \mu_{N}, p \varepsilon$ are integers. Then

$$
\begin{aligned}
\sum \lambda_{i} \phi\left(x_{i}\right) & \geq \sum \mu_{i} \phi\left(x_{i}\right)-\varepsilon \phi(a) \\
& =(1 / p)\left[\sum\left(p \mu_{i}\right) \phi\left(x_{i}\right)+(p \varepsilon) \phi(a)\right]-2 \varepsilon \phi(a) \\
& =(1 / p) \phi\left[\sum\left(p \mu_{i}\right) x_{i}+(p \varepsilon) a\right]-2 \varepsilon \phi(a) \geq-2 \varepsilon \phi(a)
\end{aligned}
$$

since

$$
\sum\left(p \mu_{i}\right) x_{i}+(p \varepsilon) a \geq p \sum \lambda_{i} x_{i} \geq 0 .
$$

We now have $\sum \lambda_{i} \phi_{i}\left(x_{i}\right) \geq-2 \varepsilon a$ for all $\varepsilon>0, \varepsilon \in \mathbb{Q}$. Hence, $\sum \lambda_{i} \phi\left(x_{i}\right)$ $\geq 0$.

(III) Finally, assume that $\phi$ is a Riesz homomorphism. We may assume that $F$ is a vector lattice.

Let $x \in E$; let $u \in F$ be a lower bound of $\left\{\bar{\phi}(y): y \in E^{+}, y \geq x\right\}$; we wish to show that $u \leq(\bar{\phi}(x))^{+}$. Take $a$ as in (I). Let $\varepsilon>0$ : we prove $u \leq(\bar{\phi}(x))^{+}+2 \varepsilon \bar{\phi}(a)$. By (I) there exist $m \in N$ and $s \in G$ such that $s / m-\varepsilon a \leq x \leq s / m+\varepsilon a$. For all $t \in G^{+}$with $t \geq s$ we have $t / m+\varepsilon a \in E^{+}$ and $t / m+\varepsilon a \geq x$, so (by the given property of $u$,) $u \leq \bar{\phi}(t / m+\varepsilon a$ ). It follows that $m(u-\varepsilon \bar{\phi}(a))$ is a lower bound of $\left\{\bar{\phi}(t): t \in G^{+}, t \geq s\right\}$. But $\phi$ is a Riesz homomorphism, so $m(u-\varepsilon \bar{\phi}(a)) \leq(\phi(s))^{+}$. This implies

$$
\begin{aligned}
u & \leq(1 / m)(\phi(s))^{+}+\varepsilon \bar{\phi}(a) \leq(\bar{\phi}(s / m-\varepsilon a))^{+} 2 \varepsilon \bar{\phi}(a) \\
& \leq(\bar{\phi}(x))^{+}+2 \varepsilon \bar{\phi}(a) .
\end{aligned}
$$

Corollary 2.4. For $i=1,2$ let $E_{i}$ be an Archimedean, directed partially ordered vector space and $G_{i}$ a subgroup of $E_{i}$ that generates $E_{i}$ as a vector space. If $G_{1}$ and $G_{2}$ are isomorphic as partially ordered groups, then $E_{1}$ and $E_{2}$ are isomorphic as partially ordered vector spaces.

Theorem 2.5. Let $E$ be a Riesz space, $D$ a cofinal linear subspace of $E$ that generates $E$ as a Riesz space. Let $\phi$ be a Riesz homomorphism of $D$ 
into a Riesz space $F$. Then $\phi$ extends uniquely to a Riesz homomorphism $\bar{\phi}: E \rightarrow F$.

Proof. As $D$ generates $E$, uniqueness is no problem. For the same reason we may assume $F$ to be Dedekind complete.

$D$ is cofinal in $E$. thus, we can define $p: E \rightarrow F$ by

$$
p(a)=\inf \{\phi(x): x \in D, x \geq a\} \quad(a \in E) .
$$

Clearly

$$
\left.p\right|_{D}=\phi .
$$

Furthermore, $p$ is a sublinear $V$-homomorphism. Indeed, the sublinearity is clear. For $a, b \in E$ we have

$$
\begin{aligned}
p(a) \vee p(b) & =\inf _{\substack{x, y \in D \\
x \geq a, y \geq b}} \phi(x) \vee \phi(y)=\inf _{\substack{x, y \in D \\
x \geq a, y \geq b}} \inf _{\substack{z \in D \\
z \in x, z \geq y}} \phi(z) \\
& =\inf _{\substack{z \in D \\
z \geq a, \quad z \geq b}} \phi(z)=\inf _{\substack{c \in E \\
c \geq a, c \geq b}} \inf _{\substack{z \in D \\
z \geq c}} \phi(z) \\
& =\inf _{\substack{c \in E \\
c \geq a, c \geq b}} p(c) .
\end{aligned}
$$

Actually, $p$ is linear (and therefore a Riesz homomorphism). To see this we consider

$$
H=\{x \in E: p(-x)=p(x)\} .
$$

$H$ is a linear subspace of $E$ containing $D$, and $p$ is linear on $H$. Let $a \in H$; if we can prove $|a| \in H$, then $H$ is a Riesz subspace of $E$, so $H=E$ and we are done.

Following the construction of 2.2 we make the V-homomorphism $q=$ $p^{-|a|}$. Then $q(|a|)=-p(-|a|)$ but also (as $q=p$ on $H$ )

$$
\begin{aligned}
q(|a|) & =q(a \vee(-a))=q(a) \vee q(-a) \\
& =p(a) \vee p(-a)=p(a \vee(-a))=p(|a|) .
\end{aligned}
$$

Hence, $|a| \in H$.

Let $E$ be a po-group and $D \subset E$ be a subset. We say that $D \subset E$ is order dense if for each $e \in E$ we have $e=\inf \{d: d \geq e$ and $d \in D\}$. In particular if $D \subset E$ is order dense then $D$ is majorizing in $E$. (In fact, our definition of order dense is the common one if we assume that $D$ is majorizing).

There is an addendum to Theorem 2.5 which says that $\bar{\phi}$ is complete if $\phi$ is a complete Riesz homomorphism and $D \subset E$ is order dense. The order denseness of $D \subset E$ is necessary in that addendum. Indeed, let $E$ be the 
Riesz space of all piecewise linear functions on $[0,1], D$ the linear span of the constant function 1 and the identity function, $F=\mathbb{R}$ and $\phi(x)=x(0)$ $(x \in D)$. $\phi$ extends to a Riesz homomorphism $E \rightarrow \mathbb{R}$, that is not complete. Our addendum follows from the next lemma.

LEMMA 2.6. Let $\phi: G \rightarrow H$ be an order preserving group homomorphism. Suppose $G_{0}$ is an order dense subgroup of $G$ such that the restriction of $\phi$ to $G_{0}$ is a complete Riesz homomorphism. Then so is $\phi$ itself.

Proof. Let $X \subset G, \inf X=0$. Let $Y=\left\{y \in G_{0}\right.$ : there is an $x \in X$ with $y \geq x\}$. If $u \in G_{0}$ is a lower bound of $Y$, then (by order denseness) $u$ is a lower bound of $X$. Hence, inf $Y=0$ in $G_{0}$. Then inf $\phi(Y)=0$ in $H$, and a fortiori inf $\phi(X)=0$ in $H$.

ADDENDUM 2.7. Let $D$ in Theorem 2.5 be order dense in $E$. If $\phi$ is a complete Riesz space homomorphism then so is $\bar{\phi}$.

By combining 2.3 and 2.5 we obtain

Theorem 2.8. Let $E$ be an Archimedean Riesz space, $G$ a directed subgroup of $E$ that generates $E$ as a Riesz space. Then every Riesz homomorphism $\phi$ of $G$ into an Archimedean Riesz space $F$ extends uniquely to a Riesz homomorphism $\bar{\phi}: E \rightarrow F$. In particular: If $E$ is an Archimedean Riesz space, generated by a linear subspace $F$ that is in its own right a Riesz space, then the identity map of $F$ extends to a Riesz homomorphism $\bar{\phi}: E \rightarrow F$.

We remark that there is an alternative way of formulating Theorem 2.8. From $[9,2.2 .11]$ one sees that every element in $E$ can be represented as $\bigwedge_{I} \bigvee_{J} x_{i j}$ where $I$ and $J$ are finite and $x_{i j} \in G$ (where $G$ and $E$ are as in Theorem 2.8). Alternatively, it thus follows from our Theorem 2.8 that $\phi$ can be extended by defining $\Lambda_{I} \bigvee_{J} \phi\left(x_{i j}\right)$ to be the image of $\Lambda_{I} \bigvee_{J} x_{i j}$.

We now introduce the enveloping Riesz space.

Theorem 2.9. There exists an (essentially) unique Archimedean Riesz space $E$ containing $G$ as a partially ordered subgroup such that

(i) $E$ is, as a Riesz space, generated by $G$;

(ii) The set $\{\lambda x: \lambda \in \mathbb{Q}, x \in G\}$ is order dense in $E$.

Proof. (I) By [2, Theorem 26, p. 312], $G$ can be embedded in a Dedekind complete $l$-group. It follows from Iwasawa's theorem [2, p. 317] that $G$ is commutative, and from [2, p. 294], that

$$
n \in \mathbb{N}=\{1,2,3, \ldots\}, \quad a \in G, \quad n a \geq 0 \Rightarrow a \geq 0 .
$$


(II) $G$ generates a divisible partially ordered group $G_{1}$ as follows. In $G \times \mathbb{N}^{*}$ we define the equivalence relation $(x, n) \sim(y, m)$ if and only if $m x=n y$. We denote the equivalence class of $(x, n)$ by $[x, n]$ (and think of it as $x / n)$. Define $G_{1}=G \times \mathbb{N}^{*} / \sim G_{1}$ is a commutative, integrally closed and directed po-group. Moreover, $G_{1}$ is divisible and the correspondence $x \mapsto[x, 1](x \in G)$ is a po-group isomorphism of $G$ onto a subgroup of $G_{1}$. (It also is a complete Riesz homomorphism). By identifying $x$ with $[x, 1]$ we obtain the inclusion $G \subset G_{1}$.

(III) Let $G_{1}^{\delta}$ be the completion by nonvoid cuts; $G_{1}^{\delta}$ is an l-group. For $\lambda \in \mathbb{Q}, \lambda>0$ the multiplication by $\lambda$ is an automorphism of $G_{1}$, extending uniquely to an automorphism of $G_{1}^{\delta}$. Thus, $G_{1}^{\delta}$ becomes a vector space over $\mathbb{Q}$. With the aid of the order completeness it is perfectly easy to define multiplication with irrational elements of $\mathbb{R}$ and to turn $G_{1}^{\delta}$ into a (Dedekind complete) Riesz space.

(IV) Let $E$ be the Riesz subspace of $G_{1}^{\delta}$ generated by $G_{1}$. The set $\{\lambda x: \lambda \in \mathbb{Q}, x \in G\}$ is just $G_{1}$. The properties (i), (ii) follow. The Riesz space $E$ in Theorem 2.9 , being unique, is denoted by $E(G)$ and we call it the enveloping Riesz space of $G$. It follows that the natural map $G \rightarrow G_{1}$ is a complete Riesz homomorphism (indeed, suppose $X \subset G$ with inf $X=0$. Let $[y, n]$ in $G_{1}$ be such that $[y, n] \leq x$ for all $x \in X$. Then $y \in G$ and $y \leq n x$ for all $x \in X$ and hence (by Corollary 1.6) $y \leq 0$. Thus inf $X=0$ in $G$ ). Also, the natural map $G \rightarrow E(G)$ is a complete Riesz homomorphism (apply Corollaries 1.2 and 1.4 ). Thus, by Corollary 1.3 we have

Proposition 2.10. The natural map $G \rightarrow E(G)$ is a complete Riesz homomorphism.

As an immediate consequence of our extension theorems we have

THeOREM 2.11. Let $\phi: G \rightarrow H$ be a Riesz homomorphism. Then there exists a unique Riesz homomorphism $\phi^{E}: E(G) \rightarrow E(H)$ making the diagram

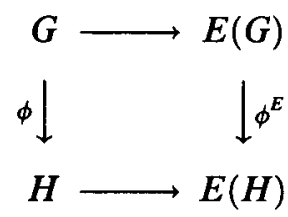

commutative. 
Proof. Let $j_{G}$ and $j_{H}$ be the natural maps $G \rightarrow E(G)$ and $H \rightarrow E(H)$. By Corollary 1.3, $j_{H} \circ \phi$ is a Riesz homomorphism. By Theorem 2.8, $j_{H} \circ \phi$ extends to a Riesz homomorphism $\phi^{E}: E(G) \rightarrow E(H)$.

\section{Riesz hulls}

We remark here that for Archimedean lattice ordered groups our results are known and contained in [6]. Bleier's main improvement [3] of Conrad's result in the lattice ordered case, is a special case of our Theorem 3.8.

Definition 3.1. An Archimedean Riesz space $E$ is called a Riesz hull of $G$ if

(i) $G$ is a partially ordered subgroup of $E$ and the identity map $G \rightarrow E$ is a complete Riesz homomorphism.

(ii) If $F$ is an Archimedean Riesz space and if $\phi: G \rightarrow F$ is a complete Riesz homomorphism, then $\phi$ extends uniquely to a complete Riesz homomorphism $\bar{\phi}: E \rightarrow F$.

The Riesz hull (if it exists) is unique:

Proposition 3.2. If $E_{1}, E_{2}$ are Riesz hulls of $G$, then there is a Riesz isomorphism of $E_{1}$ onto $E_{2}$ that extends the identity map of $G$.

Proof. The embeddings $\phi_{1}: G \rightarrow E_{1}$ and $\phi_{2}: G \rightarrow E_{2}$ induce complete Riesz homomorphism $\bar{\phi}_{1}: E_{2} \rightarrow E_{1}$ and $\phi_{2}: E_{1} \rightarrow E_{2}$. By the uniqueness part of (ii), above, we have $\bar{\phi}_{1}=\bar{\phi}_{1} \circ \bar{\phi}_{2} \circ \bar{\phi}_{1}$ and $\bar{\phi}_{2}=\bar{\phi}_{2} \circ \bar{\phi}_{1} \circ \bar{\phi}_{2}$. Then $\bar{\phi}_{1}$ and $\bar{\phi}_{2}$ are each other's inverses.

If the Riesz hull of $G$ exists we denote it by $R[G]$.

Lemma 3.3. $E(G)$ is a Riesz hull of $G$.

Proof. By Proposition 2.10 the embedding $G \rightarrow E(G)$ is a complete Riesz homomorphism. In the rest of this proof we write $E=E(G)$.

(I) We regard $G$ as a subgroup of its Dedekind completion $G^{\delta}$. Let $F$ be an Archimedean Riesz space and $\varphi: G \rightarrow F$ a complete Riesz homomorphism. We may assume $F$ is Dedekind complete (by Corollary 1.5). For $a \in G^{\delta}$ we have

$$
0=\inf \{x-y: x, y \in G, x \geq a \geq y\} \text { in } G,
$$

so that $0=\inf \{\phi(x)-\phi(y): x, y \in G, x \geq a \geq y\}$ in $F$ and $\sup \{\phi(y): y \in$ $G, y \leq a\}=\inf \{\phi(x): x \in G, x \geq a\}$ in $F$. It follows that $\phi$ extends 
uniquely to an increasing $\phi^{\delta}: G^{\delta} \rightarrow F$. This $\phi^{\delta}$ is a complete Riesz homomorphism.

(II) $\phi^{\delta}$ extends naturally to an increasing linear map $\omega: G^{\delta} \rightarrow F$. We proceed to show that $\omega$ is again a complete Riesz homomorphism.

Let $X \subset G^{\delta}, \inf X=0$. Let $u=\inf \omega(X) \in F$ and suppose $u>0$. We may assume that $X \cap G^{\delta}$ contains an element $e$. Choose $N \in \mathbb{N}$ such that $\left(N u-\phi^{\delta}(e)\right)^{+}>0$.

For every $x \in X$ there is a smallest (we put 'smallest' to avoid $A C$ ) $n \in \mathbb{N}$ with $n x \in G^{\delta}$; take $x \leq e$ in $X$ and put $x^{\prime}=\left[n(x-(1 / N) e)^{+} \wedge e\right] \in G^{\delta}$. Then

$$
\begin{aligned}
\varphi^{\delta}\left(x^{\prime}\right) & =\varphi^{\delta}\left(n\left(x-\frac{1}{N} e\right)^{+} \wedge e\right) \\
& =\varphi^{\delta}\left(n\left(x-\frac{1}{N} e\right)^{+}\right) \wedge \varphi^{\delta}(e) \\
& =n \varphi^{\delta}\left(\left(x-\frac{1}{N} e\right)^{+}\right) \wedge \omega(e) \\
& =\frac{n}{N}\left[n \omega(x)-\varphi^{\delta}(e)\right]^{+} \wedge \omega(e) \\
& \geq \frac{n}{N}\left[N u-\varphi^{\delta}(e)\right]^{+} \wedge u .
\end{aligned}
$$

As $(n / N)\left[N u-\varphi^{\delta}(e)\right]^{+} \wedge u>0$ we obtain $\inf \left\{\varphi^{\delta}\left(x^{\prime}\right): x \in X\right\}>0$ in $F$. On the other hand, $0 \leq x^{\prime} \leq N x$ and $\inf \{N x: x \in X\}=0$ in $G^{\delta}$, so $\inf \left\{x^{\prime}: x \in X\right\}=0$ in $G^{\delta}$. Contradiction.

(III) The rest is simple $G$ is order dense in $G^{\delta}$, so that $\mathbb{Q} G$ is order dense in $\mathbb{Q} G^{\delta}$. Then the restriction of $\omega$ to $\mathbb{Q} G$ is a complete Riesz homomorphism. $\mathbb{Q} G$ is order dense in $E$, so $\left.\omega\right|_{Q G}$ extends to an increasing map $\bar{\phi}: E \rightarrow F$. Then $\bar{\phi}$ is a complete Riesz homomorphism.

We now collect some consequences.

THEOREM 3.4. $G$ has a Riesz hull $R[G]$. Considered as a subset of $R[G]$, $G$ generates $R[G]$ as a Riesz space.

Corollary 3.5. Let $E$ be an Archimedean Riesz space containing $G$ as a partially ordered subgroup. Then $E$ is a Riesz hull of $G$ if and only if $E$ is generated by $G$ as a Riesz space and $\mathbb{Q} G$ is order dense in $E$.

Corollary 3.6. Let $\phi: G \rightarrow H$ be a complete Riesz homomorphism. Then there exists a unique Riesz homomorphism $\phi^{R}: R[G] \rightarrow R[H]$ such 
that the diagram

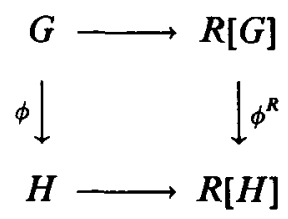

is commutative. This $\phi^{R}$ is complete.

Proof. Existence and uniqueness of $\phi^{R}$ follow from Theorem 2.8; the completeness is a consequence of Corollary $1.6(G \rightarrow R[H]$ is complete) and Theorem $3.4(R[G]$ is a Riesz hull of $G)$.

Corollary 3.7. $R[G]$ is a "smallest" Riesz space extension of $G:$ Let $E$ be an Archimedean Riesz space, $G$ a (directed) subgroup of $E$ that generates $E$ as a Riesz space. Then the identity map of $G$ extends to a surjective Riesz homomorphism $E \rightarrow R[G]$.

Proof. The natural map $G \rightarrow R[G]$ extends to a Riesz homomorphism $\phi: E \rightarrow R[G]$ according to Theorem 2.8 .

We have the following generalization of Theorem 3.4.

THEOREM 3.8. Let $G$ be a directed subgroup of an Archimedean Riesz space $E$. Then the following are equivalent.

(i) The natural map $\alpha: G \rightarrow E(G)$ extends to a Riesz isomorphism, $E \rightarrow$ $E(G)$.

(ii) $E$ is generated by $G$ as a Riesz space and $\mathbb{Q} G$ is order dense in $E$.

(iii) $E$ is generated by $G$ and $G \rightarrow E$ is a complete Riesz homomorphism.

(iv) $E$ is generated by $G$ and $G \rightarrow E$ is a Riesz homomorphism.

If $G$ is lattice ordered as well then each of (i)-(iv) is equivalent to:

(v) $E$ is generated by $G$ and $G$ is large in $E$.

(vi) $E$ is generated by $G$.

Proof. (i) $\Rightarrow$ (ii) follows from the construction of $E(G)$.

(ii) $\Rightarrow$ (iii). By Corollary 3.7, $E$ is isomorphic with $E(G)$ and thus $G \rightarrow E$ is a complete Riesz homomorphism.

(iii) $\Rightarrow$ (iv) is trivial.

(iv) $\Rightarrow$ (i). By Corollary 3.7 there is a surjective Riesz homomorphism $\alpha: E \rightarrow E(G)$ extending the natural inclusion $G \rightarrow E(G)$. By Lemma 2.3 
there exists a Riesz homomorphism $\delta: E(G) \rightarrow E$ extending the inclusion $G \subset E$. Then $\delta \circ \alpha: E \rightarrow E$ is a Riesz homomorphism $(E(G)$ and $E$ are Riesz spaces!) which is the identity on $G$. It follows that $\alpha$ is injective and thus a Riesz isomorphism.

Now assume $G$ is lattice ordered as well.

(ii) $\Rightarrow$ (v). If $h>0$ in $E$ then $h=\sup \{q: q \leq h, q \in G\}=\sup \left\{q^{+}: q \leq\right.$ $h, q \in G\}$ and thus some $q^{+} \in G$ must be nonzero and less than or equal to $h$.

(vi) $\Rightarrow$ (iv) is trivial.

\section{Examples}

EXAmple 4.1. Let $H$ be a Hilbert space, $A$ the ordered vector space of all hermitian operators in $H, A_{0}$ the linear subspace of $A$ generated by $I$ and the hermitian operators of finite rank.

Let $S=\{t \in H:\|t\|=1\}$ and let $C_{e}(S)$ be the Riesz space of all continuous functions $f: S \rightarrow \mathbb{R}$ for which $f(-t)=f(t) \quad(t \in S)$. To $a \in A$ assign $\hat{a} \in C_{e}(S): \hat{a}(t)=\langle a(t), t\rangle \quad(t \in S)$. The assignment $a \rightarrow \hat{a}$ is a bipositive linear map $A \rightarrow C_{e}(S)$.

It follows that $E(A)$ and $E\left(A_{0}\right)$ are the Riesz subspaces of $C_{e}(S)$ generated by $A$ and $A_{0}$ respectively. The order denseness of $A_{0}$ in $E\left(A_{0}\right)$ follows easily from the observation that $\widehat{A_{0}}$ is a linear subspace of $C_{e}(S)$ containing the constants and containing, for each $s \in S$, the function $t \rightarrow\langle t, s\rangle^{2}$ $(t \in S)$ that peaks at $\pm \delta$ and nowhere else. (This function is $\hat{a}$ where $a(t)=\langle t, s\rangle s)$.

EXAMPLE 4.2. Let $A$ be the ordered vector space of all hermitian $2 \times 2$ matrices. Let $\left.S=\{x, y) \in \mathbb{R}^{2} \mid x^{2}+y^{2}=1\right\}$ To $a=\left(\begin{array}{cc}\alpha & \beta \\ \beta & \delta\end{array}\right) \in A$ assign $\hat{a} \in C(S)$, defined by $\hat{a}(x, y)=(\alpha+\delta)+(\alpha-\delta) x+2 \beta y((x, y) \in S)$. Then $a \geq 0 \Leftrightarrow \alpha+\delta \geq 0$ and $\beta^{2} \leq \alpha \delta \Leftrightarrow \hat{a} \geq 0$, so $a \mapsto \hat{a}$ is bipositive. It follows that $E(A)$ is the Riesz subspace of $C(S)$ spanned by $1_{s}$ and the two coordinate functions.

EXAMPLE 4.3. Though actually we can use (see [5]) the results of this paper for (yet) another construction of the Riesz space tensor product of two Riesz spaces $E$ and $F$, what we do here is just indicate one aspect. Let $E, F$ be Archimedean Riesz spaces. Of course the vector tensor product $E \otimes F$ is an ordered vector subspace of the Riesz space tensor product $E \bar{\otimes} F$. It follows from [5] that $E \otimes F$ is order dense in $E \otimes F$. Therefore $E(E \otimes F)=E \bar{\otimes}$. 


\section{References}

[1] M. Anderson and R. Feil, Lattice ordered groups, an introduction (D. Reidel Publishing Company, 1987).

[2] G. Birkhoff, Lattice theory, 3rd edition, Colloquium Publications 25 (Amer. Math. Soc., Providence, 1984).

[3] R. D. Bleier, 'Minimal vector lattice covers', Bull. Aust. Math. Soc. 5 (1971), 331-335.

[4] G. Buskes and A. van Rooij, 'Hahn-Banach for Riesz homomorphisms', Indag. Math. A 92 (1989), 25-34.

[5] G. Buskes and A. Van Rooij, 'The Archimedean 1-group tensor product', preprint.

[6] P. F. Conrad, 'Minimal vector lattice covers', Bull. Austral. Math. Soc. 4 (1971), 35-39.

[7] P. F. Conrad, 'The hulls of representable 1-groups and $f$-rings', J. Austral. Math. Soc. (Series A) 16 (1973), 385-415.

[8] D. Fremlin, Topological Riesz spaces and measure theory (Cambridge University Press, London, 1974).

[9] G. Jameson, Ordered linear spaces, Lecture Notes in Math. 141 (Springer, Berlin, 1970).

[10] A. W. Wickstead, 'The Spectrum of an R-homomorphism', J. Austral. Math. Soc. (Series A) 23 (1977), 42-45.

[11] A. W. Wickstead, 'Relatively central operators on Archimedean vector lattices I', Proc. Roy. Irish Acad. Sect. A 80 (1980), 191-208.

The University of Mississippi

College of Liberal Arts

University, MS 38677

USA
Catholic University

Toernooiveld

6525 ED Nijmegen

The Netherlands 